\title{
Sensitivity Quantification of Remote Detection NMR and MRI
}

\author{
J. Granwehr ${ }^{a, *}$ J.A. Seeley ${ }^{a}$ \\ ${ }^{a}$ Materials Sciences Division, Lawrence Berkeley National Laboratory, and Department of Chemistry, \\ University of California, Berkeley, CA 94720, USA
}

\begin{abstract}
A sensitivity analysis of the remote detection NMR technique is presented. With remote detection, information about a sample is encoded onto a mobile sensor fluid, which facilitates a spatial separation of encoding and detection of spin magnetization. This approach can be interpreted as a two-dimensional NMR experiment, therefore the same general formalism can be used for a sensitivity analysis. Even though remote detection is a point-by-point experiment, the sensitivity does not scale unfavorably with the number of detected points compared to transient detection. It is proportional to the relative sensitivity between the remote detector and the circuit that is used for encoding. The influence of the different signal decay times is analyzed, and the distinction between spectroscopy and imaging experiments is made.
\end{abstract}

Key words: NMR, MRI, Remote detection, Sensitivity, Indirect detection, Flow

NMR remote detection, a novel technique to spatially and temporally separate encoding and detection of nuclear spin magnetization, has the potential to enhance the sensitivity of NMR spectroscopy and imaging experiments. It employs a flowing sensor medium that samples a stationary analyte of interest, which can be a liquid, a surface, a porous medium, or void space $[1,2]$. In these experiments the sensor medium, thus far hyperpolarized ${ }^{129} \mathrm{Xe}[3]$, is first introduced to a sample of interest, is encoded with information regarding that sample, and is then transferred to a different location for sensitive detection. This separation of the encoding and detection steps allows optimizing them independently. The encoding region may be configured in the most convenient way to accommodate the sample, while different conditions optimized for sensitivity are used in the detection region. This communication aims to provide a more quantitative discussion of the sensitivity achievable with this technique.

The longitudinal magnetization of the sensor medium is the property that is altered by the encoding step. An arbitrary pulse sequence can be used that is able to transfer the desired information about the stationary analyte onto longitudinal magnetization of the sensor medium.

* Corresponding author. Fax: 1-510-486-5744.

Email address: joga@waugh.cchem.berkeley.edu (J. Granwehr). 
This information can be the chemical shift of the sensor medium in contact with the sample [1]. An experiment to encode a heteronuclear spectrum from the stationary analyte to the mobile sensor using the long-range intermolecular dipole-dipole interactions was suggested as well [5]. Void-space imaging experiments have been performed with phase-encoding [2,6] as well as projection reconstruction [1]. In experiments that involve a transient evolution of transverse magnetization during encoding, one phase component after each evolution time $t_{1}^{\mathrm{r}}$ is stored with a $90^{\circ}$ pulse as longitudinal magnetization, $M_{z}$, and at the same time transfer any remaining $M_{z}$ into the $x y$ plane. Stored as polarization, the magnetization follows the external field adiabatically and is protected against dephasing due to field inhomogeneities [4], thus the sensor can travel through field gradients to the detector in a remote location without losing its information. This travel step is only restricted by the longitudinal relaxation of the sensor medium. The travel time, $t_{\text {trav }}$, has to be on the timescale of its longitudinal relaxation time $T_{1}$ or shorter, so at least part of the encoded magnetization survives. At the same time any coherent magnetization dephases, because the sensor usually experiences considerable field gradients while traveling between encoding and detection environments. Therefore this traveling step acts like a $z$ filter. When the encoded sensor medium reaches the detector, its longitudinal magnetization is read out. In other words, the indirect dimension of the resulting data set provides the information about the encoding environment. Therefore remote detection NMR is inherently a point-by-point technique.

Remote detection enables experiments at low magnetic fields without suffering the consequence of low sensitivity. This is relevant for the study of samples containing susceptibility gradients or electrically conducting components such as human subjects with metallic implants. These susceptibility gradients scale linearly with the applied magnetic field. Another example of a constraint imposed on the encoding environment is the size of the sample of study. Although a large coil may be needed to encode signal in a large object, a smaller coil can be used to detect extracted concentrated sensor [7].

A different experimental approach with remote detection is to record the arrival of the sensor medium in the detector transiently as a function of the encoded information in a time-of-flight (TOF) experiment [6,7]. While the optimum signal energy is obtained if all of the encoded sensor medium is collected and read out at once, this TOF detection provides additional information, as it allows one to correlate the TOF with the encoded information, for example by mapping the flow field of the fluid as it flows through the stationary analyte. If an inductive detector is used, such a flow profile can be measured by applying a train of $90^{\circ}$ pulses in the remote coil and recording the amplitude of the FID after each pulse. This technique complements currently used flow imaging experiments, which measure local velocity vectors and not the global flow field $[8,9]$.

Not only can the signal be measured by inductive detection, but any technique can be used that is capable of sensitively measuring the magnetization or the polarization of the sensor medium. For example, DC magnetometers such as superconducting quantum interference devices (SQUIDs) [10] or optical magnetometers [11] may be preferred at low fields. Alternatively, spin-exchange optical detection could serve as a technique that is specific to measure the polarization of noble gas sensor media. This technique is based on the transfer of spin-polarization 
from the noble gas to Rb electron spins [12]. In addition to using inductive detection with pulsed rf irradiation, it is also possible to use continuous-wave (cw) techniques. Since at least with hyperpolarized ${ }^{129} \mathrm{Xe}$ only one line with known position must be detected, this approach would not have the sensitivity disadvantage of a conventional cw experiment [13].

While in previous publications the detector was considered the main sensitivity determining parameter, the involved dephasing times influence the sensitivity as well. In general, the sensitivity of NMR remote detection can be analyzed with the same approach as a conventional two-dimensional (2D) NMR experiment $[13,14]$. This sensitivity discussion can be split into a detector independent part that only includes the relative sensitivity, $\Lambda$, between the remote detector and the circuit that is used for encoding, and a detector dependent part that discusses the ability of the different detectors to measure the polarization or the magnetic moment of the sensor spins. Note that sensitivity is not simply the signal-to-noise ratio (SNR), $\psi$, rather, it is the SNR per square root time, $\Psi=\psi / \sqrt{T_{\text {tot }}}$, where $T_{\text {tot }}$ is the total duration of the experiment. This is the quantity that will be compared in the following discussion.

A possible pulse sequence is shown in Fig. 1. With direct detection, the complex signal induced

(a)

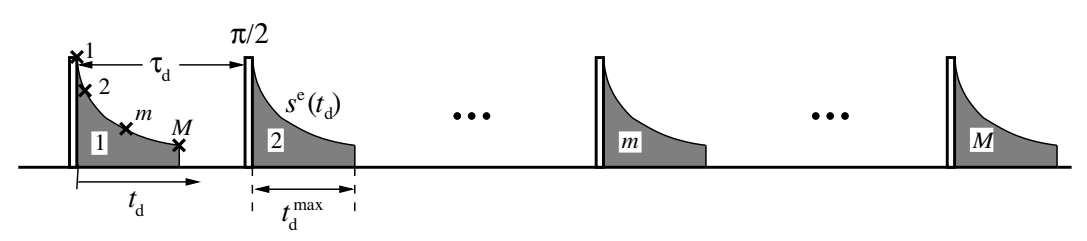

(b)

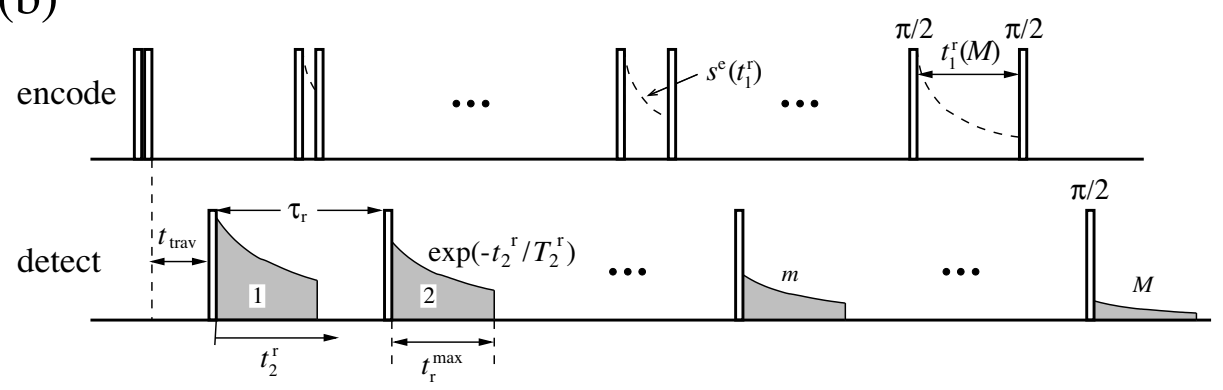

Fig. 1. Sensitivity comparison between direct (a) and remote detection (b). With direct detection, a complex FID is recorded transiently with $M$ data points, which are marked with ' $\times$ ' symbols in the first FID. Remotely, $2 M$ encoding steps are necessary to obtain the same data set, which allows one to perform $2 M$ signal averaging steps in the direct dimension in the same time. The encoding and detection steps in the remote experiment are intermingled, therefore only a time overhead corresponding to one travel time occurs. The stored magnetization of the $m$ th encoding step corresponds to the $m$ th data point with direct detection and marks the magnetization at the beginning of the remote detection. The sensitivity is proportional to the total area under all the FIDs in both cases.

in the encoding coil by the FID of a single resonance can be described as

$$
s\left(t_{\mathrm{d}}\right)=s^{\mathrm{e}}\left(t_{\mathrm{d}}\right) \exp \left(i \Omega t_{\mathrm{d}}\right)
$$

with the envelope function, $s^{\mathrm{e}}\left(t_{\mathrm{d}}\right)$, and the offset precessional frequency, $\Omega$. In the following 
calculations, it is assumed that $s^{\mathrm{e}}$ represents an exponential decay. The signal is sampled by $M$ sampling points between 0 and $t_{\mathrm{d}}^{\max }$, spaced by the dwell time $\Delta t$. Assuming white random noise limited by an analog filter with a bandwidth

$$
\Delta f=\frac{1}{\Delta t}=\frac{M}{t_{\mathrm{d}}^{\max }}
$$

that cuts off frequencies above the Nyquist frequency of the sampling process, the r.m.s. noise amplitude is

$$
\sigma_{\mathrm{d}}=\sqrt{\Delta f} \rho_{\mathrm{d}}=\sqrt{\frac{M}{t_{\mathrm{d}}^{\max }}} \rho_{\mathrm{d}},
$$

where $\rho_{n}$ is the square root of the frequency-independent power spectral density. Because $M$ data points are recorded during $\tau_{\mathrm{d}}$, which is the repetition time, we can say that the time it takes to acquire one data point is $t_{\mathrm{d}}^{\mathrm{aq}}=\tau_{\mathrm{d}} / M$. Thus we get a sensitivity for one data point of

$$
\Psi_{\mathrm{d}}=\left|\frac{s\left(t_{\mathrm{d}}\right)}{\sigma_{\mathrm{d}} \sqrt{t_{\mathrm{aq}}}}\right|=\frac{s^{\mathrm{e}}\left(t_{\mathrm{d}}\right)}{\rho_{\mathrm{d}}} \sqrt{\frac{t_{\mathrm{d}}^{\max }}{\tau_{\mathrm{d}}}} .
$$

$t_{\mathrm{d}}^{\max } / \tau_{\mathrm{d}}$ represents the duty cycle of this experiment.

To determine the sensitivity of one point of a remotely detected FID, the signal is described by

$$
s\left(t_{1}^{\mathrm{r}}, t_{2}^{\mathrm{r}}\right)=s^{\mathrm{e}}\left(t_{1}^{\mathrm{r}}\right) \exp \left(i \Omega_{2} t_{2}^{\mathrm{r}}-\frac{t_{2}^{\mathrm{r}}}{T_{2}^{\mathrm{r}}}\right)\left[\cos \left(\Omega t_{1}^{\mathrm{r}}\right)+j \sin \left(\Omega t_{1}^{\mathrm{r}}\right)\right]
$$

where we assume that the travel time $t_{\text {trav }}$ of the sensor medium is much shorter than its $T_{1}$. This signal oscillates at $\Omega_{2}$ and decays with a time constant $T_{2}^{\mathrm{r}}$ during remote detection. The imaginary units $i$ and $j$ are independent, equivalent to a hypercomplex encoding scheme [13]. The information encoded pointwise along $t_{1}^{\mathrm{r}}$ corresponds to the signal described by Eq. (1). Since only one phase component of the transverse magnetization can be stored at the end of each encoding period, the phase of the signal is lost between encoding and detection. Two encoding steps are necessary to map out one point of a complex FID, e.g. by applying the storage pulse along $-y$ and $x$ in two subsequent encoding steps to encode $M_{x}$ and $M_{y}$, respectively.

To optimize the sensitivity of a spectrum, the time-domain signal may be multiplied with a weighting function $h(t)$. Because the remotely detected signal provides merely an amplitude value for each encoding step, it is reasonable to use a matched weighting function to maximizes the sensitivity, $h_{m}\left(t_{2}^{\mathrm{r}}\right)=\exp \left(-t_{2}^{\mathrm{r}} / T_{2}^{\mathrm{r}}\right)$. The application of a matched filter results in a time constant $T_{2}^{\mathrm{r}} / 2$ of the signal. This filter will only be applied in the $t_{2}^{\mathrm{r}}$ dimension of the remote experiment for this treatment. Since it is not applied in the $t_{1}^{\mathrm{r}}$ dimension, the dimension analogous to the $t_{\mathrm{d}}$ dimension we are comparing it to, we do not need to apply a filter to the directly detected signal of Eq. (1) for a fair comparison. Each complex data point in the $t_{1}^{\mathrm{r}}$ dimension is recorded with a sensitivity 


$$
\begin{aligned}
\Psi_{\mathrm{r}} & =\left|\frac{S\left(t_{1}^{\mathrm{r}}\right)}{\sigma_{N} \sqrt{t_{\mathrm{r}}^{\mathrm{aq}}}}\right|_{\text {matched }}=\frac{\left|s\left(t_{1}^{\mathrm{r}}\right)\right|}{\rho_{\mathrm{r}}} \sqrt{\left[\frac{t_{\mathrm{r}}^{\max }}{t_{\mathrm{r}}^{\mathrm{aq}}}\right]\left[\frac{1}{t_{\mathrm{r}}^{\max }} \int_{0}^{t_{\mathrm{r}}^{\max }}\left[\exp \left(-\frac{t_{2}^{\mathrm{r}}}{T_{2}^{\mathrm{r}}}\right)\right]^{2} \mathrm{~d} t_{\mathrm{r}}\right]} \\
& =\frac{s^{\mathrm{e}\left(t_{1}^{\mathrm{r}}\right)}}{\rho_{\mathrm{r}}} \sqrt{\frac{T_{2}^{\mathrm{r}}}{2\left(2 \tau_{\mathrm{r}}+t_{\text {trav }} / M\right)}\left(1-\exp \left(-\frac{2 t_{\mathrm{r}}^{\max }}{T_{2}^{\mathrm{r}}}\right)\right)} .
\end{aligned}
$$

If encoding and detection are intermingled, $t_{\text {trav }}$ contributes only once to the total experiment time, and $t_{\mathrm{r}}^{\mathrm{aq}}=2 \tau_{\mathrm{r}}+t_{\text {trav }} / M$. For a typical number of data points $M$ on the order of at least 100 , we can neglect this additional contribution to the total experiment time, as $t_{\text {trav }} / M \ll \tau_{\text {r }}$. The relative sensitivity between remote and direct detection then is

$$
\left[\frac{\Psi_{\mathrm{r}}}{\Psi_{\mathrm{d}}}\right]_{\mathrm{NMR}}=\frac{\Lambda}{\sqrt{2}} \sqrt{\left[\frac{T_{2}^{\mathrm{r}} / 2 \tau_{\mathrm{r}}}{t_{\mathrm{d}}^{\max } / \tau_{\mathrm{d}}}\right]\left[1-\exp \left(-\frac{2 t_{\mathrm{r}}^{\max }}{T_{2}^{\mathrm{r}}}\right)\right]},
$$

where $\mathrm{d}$ and $\mathrm{r}$ denote the corresponding parameters of the direct and the remote experiment, respectively. $\Lambda$ represents the relative SNR between remote detector and encoding circuit as obtained with an identical sample and equal experimental parameters. The factor $1 / \sqrt{2}$ is because two cycles are required to obtain the complex signal with remote detection. Equation (7) is not dependent on $M$; the sensitivity ratio of remote-to-direct detection is independent of the number of points in the spectrum even though adding more points increases the experimental time. Remote detection can be considered to be an experiment in which the signal averaging is done in the direct dimension with a certain time constant, which is $T_{2}^{\mathrm{r}} / 2$ in this case. The interferogram is collected in the indirect dimension. In contrast, in an experiment with direct detection the interferogram is recorded transiently, and signal averaging to improve the SNR is done in an indirect dimension. Each trace has the same signal amplitude, and therefore the time constant, analogous to that with remote detection, is infinitely long. Depending on the value of $T_{2}^{\mathrm{r}}$, Eq. (7) can be simplified. Assuming that $\tau_{\mathrm{d}}=\tau_{\mathrm{r}}$, we get

$$
\begin{array}{ll}
{\left[\frac{\Psi_{\mathrm{r}}}{\Psi_{\mathrm{d}}}\right]_{\mathrm{NMR}} \approx \frac{\Lambda}{\sqrt{2}} \sqrt{\frac{T_{2}^{\mathrm{r}}}{2 t_{\mathrm{d}}^{\max }}} \quad \text { if } t_{\mathrm{r}}^{\max }>T_{2}^{\mathrm{r}}} \\
{\left[\frac{\Psi_{\mathrm{r}}}{\Psi_{\mathrm{d}}}\right]_{\mathrm{NMR}} \approx \frac{\Lambda}{\sqrt{2}}} & \text { if } t_{\mathrm{r}}^{\max } \ll T_{2}^{\mathrm{r}} .
\end{array}
$$

The first approximation is realistic in continuous flow experiments. Immediately after each detection pulse, the fluid starts to flow out of the detection volume, thus shortening $T_{2}^{\mathrm{r}}$. The second approximation may be reasonable in stopped-flow mode in a well-shimmed detection environment. This is the ideal case where the sensitivity ratio depends, except for the factor $\sqrt{2}$, only on $\Lambda$.

The situation is different in an experiment where the transient dimension of the direct detection experiment itself measures only the amplitude and the phase as opposed to the time evolution of the signal. This is the case for example in an MRI experiment with phase encoding in all three 
dimensions. Here, it is the $1 \mathrm{D}$ sensitivity that has to be compared between remote and direct detection, because the remote experiment does not sample a transient signal point-by-point. Using the same formalism as for Eq. (7), this yields

$$
\begin{aligned}
& {\left[\frac{\Psi_{\mathrm{r}}}{\Psi_{\mathrm{d}}}\right]_{\mathrm{MRI}} }=\frac{\Lambda}{\sqrt{2}} \sqrt{\left[\frac{\frac{t_{\mathrm{r}}^{\max }}{\tau_{\mathrm{r}}}}{\frac{t_{\mathrm{d}}^{\max }}{\tau_{\mathrm{d}}}}\right] \frac{\frac{1}{t_{\mathrm{r}}^{\max }} \int_{0}^{t_{\mathrm{r}}^{\max }}\left[\exp \left(-\frac{t_{2}^{\mathrm{r}}}{T_{2}^{\mathrm{r}}}\right)\right]^{2} \mathrm{~d} t_{2}^{\mathrm{r}}}{\frac{1}{t_{\mathrm{d}}^{\max }} \int_{0}^{\max }\left[\exp \left(-\frac{t_{\mathrm{d}}}{T_{2}^{\mathrm{d}}}\right)\right]^{2} \mathrm{~d} t_{\mathrm{d}}}} \\
&=\frac{\Lambda}{\sqrt{2}} \sqrt{\frac{\frac{T_{2}^{\mathrm{r}}}{\tau_{\mathrm{r}}}\left(1-\exp \left(-\frac{2 t_{\mathrm{r}}^{\max }}{T_{2}^{\mathrm{r}}}\right)\right)}{\frac{T_{2}^{\mathrm{d}}}{\tau_{\mathrm{d}}}\left(1-\exp \left(-\frac{2 t_{\mathrm{d}}^{\max }}{T_{2}^{\mathrm{d}}}\right)\right)} \approx \frac{\Lambda}{\sqrt{2}} \sqrt{\frac{T_{2}^{\mathrm{r}}}{T_{2}^{\mathrm{d}}}}} .
\end{aligned}
$$

For the approximation it was assumed that $\tau_{\mathrm{d}}=\tau_{\mathrm{r}}$ and $t_{\mathrm{r}}^{\max }>T_{2}^{\mathrm{r}}, t_{\mathrm{d}}^{\max }>T_{2}^{\mathrm{d}}$. A comparison between Eqn. (7) and (10) shows that the potential signal advantage of remote detection in the case of imaging experiments is considerably larger than for spectroscopy experiments because for imaging, the bandwidth of the direct experiment is determined by the signal decay time and not by the signal averaging. This is particularly important for samples with large susceptibility gradients, which cause fast dephasing of the directly detected transverse magnetization. For example, the signal decay of ${ }^{129} \mathrm{Xe}$ in a Bentheimer sandstone rock [6] was on the order of $1 \mathrm{~ms}$, while in the remote environment the decay was mainly determined by the outflow of the gas from the detection volume, causing an apparent decay time on the order of $30 \mathrm{~ms}$.

In the above calculations it was assumed that all of the sensor medium gets fully regenerated between different repetitions of the experiment, which is reasonable because it is not a $T_{1}$ decay that determines this "relaxation", but a flow that forces the encoded sensor to move ahead. Another factor that has been left out is longitudinal relaxation of the sensor medium between encoding and detection. This simply causes the remote sensitivity to be multiplied by a factor $\exp \left(-t_{\text {trav }} / T_{1}\right)$, which is close to unity for ${ }^{129} \mathrm{Xe}$, but can be considerably smaller for a different sensor medium. Another aspect that has not been covered is that the sensor medium could disperse between encoding and detection, and thereby be diluted with unencoded fluid. This would require either a detector with a bigger active volume so still all the encoded fluid can be read out in one experiment, or multiple detection steps to catch all the encoded gas. In the case of a spectroscopy experiment without spatial dependence of the encoded information, it is not necessary to gather all the encoded fluid, and the signal would just be scaled proportionally to the amount of encoded fluid in the detector. If encoding steps are close enough to each other that a significant fraction of fluid gets encoded more than once, similar artifacts as with short repetition times in conventional 2D spectra can be expected [15]. If spatial information is encoded, it is required that the entire encoded sensor medium is detected. If it is diluted, the sensitivity is reduced, but the image can still be reconstructed accurately. However, if some of the encoded fluid remains undetected, the image will be weighted unevenly [2]. 
Remote detection is affected by multiplicative noise (or $t_{1}$ noise) $[5,16]$, caused by fluctuations of the signal-inducing quantity, for example due to instabilities of the polarization and flow rate of the sensor medium or the spectrometer and its environment. A larger signal contributes a proportionately higher noise figure. This is a problem that is minor in a transiently detected dimension, where this kind of noise mainly appears as a fluctuation of the phase and amplitude of the peaks. However in experiments with point-by-point detection the correlation of the noise between adjacent data points is lost, resulting in a frequency-independent noise figure (white noise). In addition, the noise across the entire spectrum will be determined by the most intense peaks. Thus less intense peaks are doomed to compete with the large noise figure from the intense peaks, sometimes rendering the smaller peaks undetectable. This multiplicative noise poses an upper limit to the SNR that can be obtained. If this limit is reached, increasing the signal does not improve the sensitivity anymore, as the noise increases accordingly.

While the optimum sensitivity with remote detection is obtained if the volume of the void space inside the analyte, $V_{s}$, and the detection volume, $V_{d}$, are matched, a TOF experiment requires sacrificing some of the sensitivity by reducing $V_{d}$ and applying multiple detection steps after each encoding step. This provides an encoded data set for each sampled time. If, for example, an image is encoded, the fluid from different locations reaches the detector at different times - the farther it was encoded from the detector, the later it usually arrives there. A partial image is obtained for each TOF, corresponding to the fluid a certain time away from reaching the detector. Comparing images at different TOF allows to follow the fluid as it crosses the sample. To avoid artifacts and to maintain good sensitivity in a TOF experiment, the time between subsequent detection pulses should correspond to the time it takes the fluid to flow through the detection volume. Let us assume that with TOF detection, $\tau_{\mathrm{r}}$ is split into $n$ detection steps, as shown in Fig. 2a. The acquisition time for each detection step shall be $t_{\mathrm{t}}^{\max }=\left(\tau_{\mathrm{r}}-t_{1}^{\mathrm{r}}\right) / n \approx \tau_{\mathrm{r}} / n$. The approximation assumes $t_{1}^{\mathrm{r}} \ll \tau_{\mathrm{r}}$. If $T_{2}^{\mathrm{r}}>t_{\mathrm{t}}^{\max }$, the signal decay time with stroboscopic detection, $T_{2}^{\mathrm{t}}$, is effectively determined by the time of the outflow of the fluid from the detection volume. Setting $T_{2}^{\mathrm{t}}$ to half of the time it takes the fluid to flow through the detection volume, $T_{2}^{\mathrm{t}}=\tau_{\mathrm{r}} / 2 n$, is a reasonable estimate. This allows one to calculate the relative sensitivity between one acquisition in a TOF experiment and single-step remote detection, where all the encoded fluid is detected in a single event, by using Eq. (6) with the respective parameters for the two experiments. The total time for each repetition is $\tau_{\mathrm{r}}$ in both cases, therefore the relative duty cycle is $T_{2}^{\mathrm{t}} / T_{2}^{\mathrm{r}}$, and one obtains

$$
\frac{\Psi_{\mathrm{t}}}{\Psi_{\mathrm{r}}}=\lambda \sqrt{\left[\frac{T_{2}^{\mathrm{t}}}{T_{2}^{\mathrm{r}}}\right]\left[1-\exp \left(-\frac{2 t_{\mathrm{r}}^{\max }}{T_{2}^{\mathrm{r}}}\right)\right]^{-1}} \approx \lambda \sqrt{\frac{T_{2}^{\mathrm{t}}}{T_{2}^{\mathrm{r}}}}=\lambda \sqrt{\frac{\tau_{\mathrm{r}}}{2 n T_{2}^{\mathrm{r}}}}
$$

The index $t$ denotes the respective parameters with TOF detection. $\lambda$ is the relative sensitivity between the detectors with the small and the big detection volume. For the approximation, $t_{\mathrm{r}}^{\max }>T_{2}^{\mathrm{r}}$ was assumed, and for the TOF experiment, $1-\exp \left(-2 t_{\mathrm{t}}^{\max } / T_{2}^{\mathrm{t}}\right) \approx 1$ was used. Eq. (11) gives an upper limit estimate of the sensitivity ratio of a partial to a single-step image. To get the full image with TOF detection, all $n$ partial images must be added up, which causes the noise to scale with $\sqrt{n}$ and degrades $\Psi_{\mathrm{t}} / \Psi_{\mathrm{r}}$ accordingly. Therefore, if the coil-size dependence of $\lambda$ is disregarded, the sensitivity to obtain the full image with TOF detection scales with $1 / n$ 
(a)

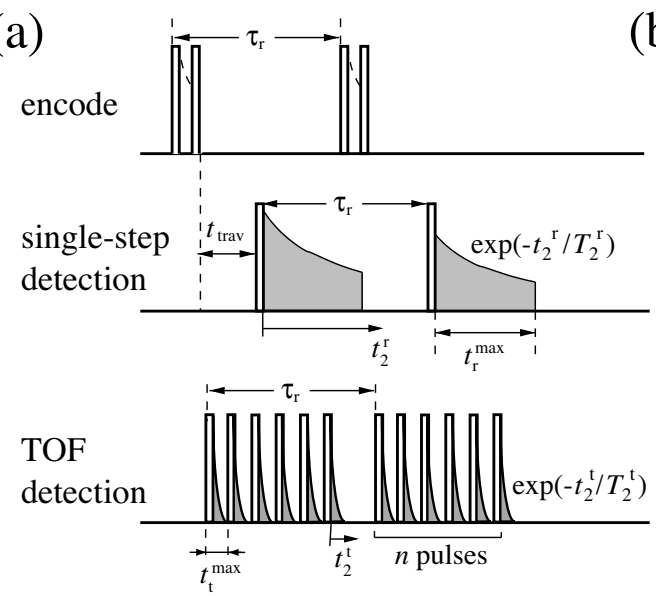

(b)

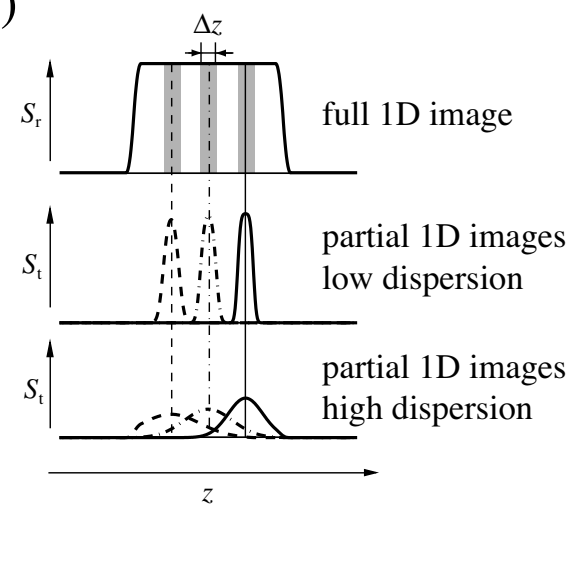

Fig. 2. Remote detection experiment with single-step and with TOF detection. (a) Timing of the experiments. The encoding sequence is independent of the detection method (upper). Single-step detection uses a single pulse, timed such that all of the encoded fluid is in the detection volume (middle). With TOF detection, a train of $n$ pulses is used to stroboscopically resolve the TOF of the sensor fluid from the encoding location to the detector (lower). (b) Illustration of the signal in a single-step and a partial 1D image. (upper) With single-step detection or if all the partial images with TOF detection are added, the full image is obtained. The gray areas correspond to partial images if there were no dispersion at all. (middle) The part of the image acquired at a particular TOF has the same amplitude as the corresponding slice of the full image if the spatial dispersion width is lower than the resolution of the image, $\Delta z$. (lower) If the dispersion width is larger than $\Delta z$, the signal at each location gets spread across multiple TOF values, and each amplitude is significantly reduced. $\lambda$ in Eq. (11) must be scaled accordingly.

compared to single-step detection. On the other hand, if spatial encoding is done in the flow direction of the sensor medium and the dispersion of the fluid between encoding location and detector is low, it is possible that the sinusoidal pattern of the longitudinal magnetization after the storage pulse is preserved during the flow. Therefore partial images can have almost the full local amplitude, and their sensitivity is given by Eq. (11). If the spatial dispersion of the fluid is larger than the image resolution, the signal of partial images is reduced, and $\Psi_{\mathrm{t}} / \Psi_{\mathrm{r}}$ is scaled proportionally. This is illustrated in Fig. 2b.

The above discussion does not take into account that a smaller detector can be made more sensitive than a large one. Detailed discussions can be found in the literature $[17,18]$. To give only a rough estimate for $\lambda$ and also $\Lambda$ if an inductive detector is used, we can use the SNR equation

$$
\psi_{\mathrm{LCR}}=K \eta M_{0} V_{c} \sqrt{\frac{Q \omega_{0}}{V_{c} T \Delta f}}=\frac{K m_{0}}{2} \sqrt{\frac{Q \omega_{0}}{V_{c} T \Delta f}},
$$

where $K$ is a numerical factor which depends on coil geometry, the noise figure of the preamplifier, and also takes into account various physical constants. $M_{0}$ is the nuclear magnetization, $V_{c}$ is the volume of the coil, $\eta \approx V_{d} / 2 V_{c}$ is the filling factor of the sample in the detection coil, $Q$ is the quality factor and $\omega_{0}$ is the resonance frequency of the $\mathrm{rf}$ circuit, $T$ is the temperature of the probe, and $\Delta f$ is the detection bandwidth $[17,19] . \Delta f$ in a pulsed NMR experiment is 
inversely proportional to $T_{2}$ and has already been included in Eqn. (7) and (10). $m_{0}=M_{0} V_{d}$ is the net magnetic moment of the spins inside the coil volume, which is transported without loss from the encoding to the detection location in an ideal remote experiment. $\lambda$ can be estimated if we note that the only factors that change by scaling the coil are $Q$ and $V_{c}$. In the ideal case, the small volume is contained $n$ times in the big volume, $V_{c}^{\mathrm{t}} / V_{c}^{\mathrm{r}}=1 / n$, therefore

$$
\lambda=\sqrt{\frac{Q_{\mathrm{t}} / V_{c}^{\mathrm{t}}}{Q_{\mathrm{r}} / V_{c}^{\mathrm{r}}}}=\sqrt{n \frac{Q_{\mathrm{t}}}{Q_{\mathrm{r}}}} .
$$

$Q$ is typically lower for smaller coils [18], but as a very rough upper limit estimate, one gets back a factor of $\sqrt{n}$ in sensitivity by using a smaller coil in the TOF experiments. Therefore, partial images can be obtained with a minimum loss in sensitivity compared to a full image using a detector with a matched volume. If the spatial resolution in the flow direction is coarse enough that images of adjacent TOF values cover a distinct fraction of the sample volume, $\Psi_{\mathrm{t}} / \Psi_{\mathrm{r}}$ may even be independent of $n$.

To experimentally determine the relative sensitivity between two coils, the principle of reciprocity, which states that the SNR is proportional to the $B_{1}$ field of a coil induced by a unit current at the sample location, can be very helpful. Since the duration of a $90^{\circ}$ pulse, $t_{90}$, is inversely proportional to $B_{1}$, one can simply use

$$
\Lambda=\frac{t_{90}^{\mathrm{d}}}{t_{90}^{\mathrm{r}}}=\sqrt{\frac{K_{\mathrm{r}} Q_{\mathrm{r}} \omega_{0}^{\mathrm{r}} / \tau_{\mathrm{r}} V_{c}^{\mathrm{r}}}{K_{\mathrm{d}} Q_{\mathrm{d}} \omega_{0}^{\mathrm{d}} / \tau_{\mathrm{d}} V_{c}^{\mathrm{d}}}} .
$$

It was assumed that $m_{0}$ does not change between encoding and detection. The SNR in a remote experiment can be enhanced by optimizing the $V_{c}^{\mathrm{r}}, Q_{\mathrm{r}}, \omega_{0}^{\mathrm{r}}, \tau_{\mathrm{r}}$, or the coil geometry $\left(K_{\mathrm{r}}\right)$. The flexibility is increased by considering the option to use alternative detectors. Magnetometers, for example, offer the possibility to measure the longitudinal magnetization, which can have a lifetime of tens of minutes in the case of a noble gas as sensor medium, even at low field [11]. Therefore the time constant to measure one data point can be adjusted to the required sensitivity by changing the duration of the acquisition, which could be useful in experiments that inherently allow for very few encoding steps only.

In conclusion, it was shown that remote detection offers a valuable alternative for sensitivity enhancements of NMR experiments with samples that cannot be tailored to fit the restrictive requirements of high-resolution setups. Especially imaging experiments of porous samples have a large potential for sensitivity improvements, while spectroscopic experiments are more limited and typically require a detector that is at least an order of magnitude more sensitive to see a noticeable sensitivity improvement. And TOF experiments, if set up properly, do not suffer a large sensitivity penalty by splitting the detection into multiple steps, while gaining an additional transient dimension without increasing the total experiment time. 


\section{Acknowledgments}

We are grateful to Alex Pines for his encouragement, support, and critical validation of this work. We would like to thank Song-I Han, Christian Hilty, and Elad Harel for helpful discussions. This work was supported by the Director, Office of Science, Office of Basic Energy Sciences, Materials Sciences and Nuclear Science Divisions, of the U.S. Department of Energy under contract DE-AC03-76SF00098. J.G. gratefully acknowledges the Swiss National Science Foundation for support through a postdoctoral fellowship.

\section{References}

[1] A.J. Moulé, M.M. Spence, S. Han, J.A. Seeley, K.L. Pierce, S.K. Saxena, A. Pines, Amplification of xenon NMR and MRI by remote detection, Proc. Natl. Acad. Sci. USA 100 (2003) 9122-9127.

[2] J.A. Seeley, S. Han, A. Pines, Remotely detected high-field MRI of porous samples, J. Magn. Reson. 167 (2004) 282-290.

[3] T.G. Walker, W. Happer, Spin-exchange optical pumping of noble-gas nuclei, Rev. Mod. Phys. 69 (1997) 629-642.

[4] A. Abragam, Principles of Nuclear Magnetism, Oxford University Press, Oxford, 1961.

[5] J. Granwehr, J.T. Urban, A.H. Trabesinger, A. Pines, NMR detection using laser-polarized xenon as a dipolar sensor, J. Magn. Reson. 176 (2005) 125-139.

[6] J. Granwehr, E. Harel, S. Han, S. Garcia, A. Pines, P.N. Sen, Y.-Q. Song, Time-of-flight flow imaging using NMR remote detection, Phys. Rev. Lett. 95 (2005) 075503.

[7] C. Hilty, E.E. McDonnell, J. Granwehr, K.L. Pierce, S. Han, A. Pines, Microfluidic gas-flow profiling using remote-detection NMR, Proc. Natl. Acad. Sci. USA 102 (2005) 14960-14963.

[8] E. Fukushima, Nuclear magnetic resonance as a tool to study flow, Annu. Rev. Fluid Mech. 31 (1999) 95-123.

[9] J.D. Seymour, P.T. Callaghan, Generalized approach to NMR analysis of flow and dispersion in porous media, AIChE J. 43 (1997) 2096-2111.

[10] Y.S. Greenberg, Application of superconducting quantum interference devices to nuclear magnetic resonance, Rev. Mod. Phys. 70 (1998) 175-222.

[11] V.V. Yashchuk, J. Granwehr, D.F. Kimball, S.M. Rochester, A.H. Trabesinger, J.T. Urban, D. Budker, A. Pines, Atomic magnetometry for detection of nuclear magnetization, Phys. Rev. Lett. 93 (2004) 160801.

[12] D. Raftery, H.W. Long, D. Shykind, P.J. Grandinetti, A. Pines, Multiple-pulse nuclear magnetic resonance of optically pumped xenon in a low magnetic field, Phys. Rev. A 50 (1994) 567-574.

[13] R.R. Ernst, G. Bodenhausen, A. Wokaun, Principles of Nuclear Magnetic Resonance in One and Two Dimensions, Clarendon Press, Oxford, 1987. 
[14] M.H. Levitt, G. Bodenhausen, R.R. Ernst, Sensitivity of Two-Dimensional Spectra, J. Magn. Reson. 58 (1984) 462-472.

[15] N. Murali, A. Kumar, Multiple-quantum artifacts in single-quantum two-dimensional correlated NMR spectra of strongly coupled spins, Chem. Phys. Lett. 128 (1986) 58-61.

[16] A.F. Mehlkopf, D. Korbee, T.A. Tiggelman, R. Freeman, Sourc es of $t_{1}$ noise in two-dimensional NMR, J. Magn. Reson. 58 (1984) 315-323.

[17] D.I. Hoult, R.E. Richards, The signal-to-noise ratio of the nuclear magnetic resonance experiment, J. Magn. Reson. 24 (1976) 71-85.

[18] D.L. Olson, T.L. Peck, A.G. Webb, R.L. Magin, J.V. Sweedler, High-resolution microcoil ${ }^{1}$ H-NMR for mass-limited, nanoliter-volume samples, Science 270 (1995) 1967-1970.

[19] R. Freeman, A Handbook of Nuclear Magnetic Resonance, $2^{\text {nd }}$ ed., Longman, Singapore, 1997. 